\title{
Modulation of apoptosis by the cyclin-dependent kinase inhibitor $\mathbf{p} 27^{\text {Kip1 }}$
}

\author{
Keiju Hiromura, ${ }^{1}$ Jeffrey W Pippin, ${ }^{1}$ Matthew L. Fero, ${ }^{2}$ James M. Roberts, ${ }^{2}$ \\ and Stuart J. Shankland ${ }^{1}$

\begin{abstract}
${ }^{1}$ Department of Medicine, Division of Nephrology, University of Washington School of Medicine, Seattle, Washington 98195-6521, USA

${ }^{2}$ Department of Basic Sciences and Howard Hughes Medical Institute, Fred Hutchinson Cancer Research Center, Seattle, Washington 98105-6521, USA
\end{abstract} \\ Address correspondence to: Stuart J. Shankland, University of Washington Medical Center, Division of Nephrology, \\ Box 356521, 1959 NE Pacific Avenue, BB1269, Seattle, Washington 98195-6521, USA. Phone: (206) 543-3792; \\ Fax: (206) 685-8661; E-mail: stuartjs@u.washington.edu
}

Received for publication October 8, 1998, and accepted in revised form January 11, 1999.

\begin{abstract}
Proliferation and apoptosis are increased in many types of inflammatory diseases. A role for the cyclin kinase inhibitor $\mathrm{p} 27^{\mathrm{Kip} 1} \mathrm{(p27)}$ in limiting proliferation has been shown. In this study, we show that $\mathrm{p} 27^{-/-}$ mesangial cells and fibroblasts have strikingly elevated rates of apoptosis, not proliferation, when deprived of growth factors. Apoptosis was rescued by restoration of p27 expression. Cyclin A-cyclindependent kinase 2 (CDK2) activity, but not cyclin E-CDK2 activity, was increased in serum-starved p27-/- cells, and decreasing CDK2 activity, either pharmacologically (Roscovitine) or by a dominant-negative mutant, inhibited apoptosis. Our results show that a new biological function for the CDK inhibitor p27 is protection of cells from apoptosis by constraining CDK2 activity. These results suggest that CDK inhibitors are necessary for coordinating the cell cycle and cell-death programs so that cell viability is maintained during exit from the cell cycle.
\end{abstract}

J. Clin. Invest. 103:597-604 (1999)

\section{Introduction}

The role of cyclin kinase inhibitors such as $\mathrm{p}^{27^{\mathrm{Kip}} 1}$ ( $\mathrm{p} 27$ ) in the cell cycle has been an area of intense study as it relates to proliferation and differentiation (1-3). Most studies suggest that proliferation requires a decrease in p27 levels, which permits cyclin-dependent kinase 2 (CDK2) activity to be generated, allowing the cell to enter the $\mathrm{S}$ phase, followed by completion of the cell cycle (4). Overexpression of p27 inhibits proliferation (5). Growth factor-induced mesangial cell proliferation in vitro is associated with decreased p27 levels (6) and increased CDK2 activity (7), and a further reduction in p27 levels with antisense augments this proliferative response (6). Mesangial cell proliferation in vivo after immune-mediated injury in experimental glomerulonephritis (Thy1 model) is also associated with decreased p27 levels (8), and proliferation is increased in p27-/- mice with glomerulonephritis compared with nephritic $\mathrm{p}^{27^{+/+}}$mice (9). In contrast, immune-mediated injury to the visceral glomerular epithelial cell (podocyte) is associated with increased p27 levels, which coincide with little if any proliferation (10). These studies show that one function of p27 is to determine the proliferative threshold in renal and nonrenal cells.

Recently, however, an increased CDK2 activity has also been associated with programmed cell death (apoptosis) (11-15). Apoptosis is a physiological form of programmed cell death that is increased in renal and nonrenal diseases and allows an organism to dispose of unwanted or defective cells $(16,17)$. In each organ, cell number is determined by a balance of proliferation and apoptosis. Thus, apop- tosis is found primarily in proliferating tissues, and apoptosis may be critical in the resolution phase of inflammatory disease such as glomerulonephritis (18). Apoptosis can be triggered by a wide variety of stimuli, and multiple pathways exist for the induction of apoptosis.

Although the role of CDK p27 in the proliferative response is established, it is not known what role p27 plays in apoptosis or in determining the fate of cells as they progress through the cell cycle. In this study, we provide novel evidence that activation of CDK2 generated by the absence of p27 allows cells to enter the cell cycle only in the presence of growth factors. In the absence of growth factors, a p27-mediated increase in CDK2 activity leads to apoptosis. Thus, p27, in conjunction with the presence or absence of a complete mitogenic signal, coordinates the final outcome of proliferation or death of the cell.

\section{Methods}

Cell culture. Mesangial cells were isolated from Sprague-Dawley rats (6) and mesangial cells and fibroblasts grown from $\mathrm{p} 27^{-/-}$and p $27^{+/+}$mice (19) were used in this study; Rat-1 fibroblasts were supplied by D.M. Hockenbery (Fred Huthinson Cancer Research Center, Seattle, Washington). To lower p27 in rat mesangial cells and rat fibroblasts, cells were transfected with $1 \mathrm{nM}$ of p27 antisense oligodeoxynucleotides (gift of M. Flanagan Gilead Scientific Inc., Foster City, California, USA) and were complexed with 1 $\mu \mathrm{g} / \mathrm{ml}$ cationic liposome (GS2888; Gilead Scientific Inc.) as reported previously $(6,20)$. Controls included nontransfected cells and cells transfected with mismatch oligodeoxynucleotides.

Inducing apoptosis. Mouse and rat mesangial cells and fibroblasts were plated at a density of $2 \times 10^{4}$ cells $/ \mathrm{cm}^{2}$ in growth media (DMEM [Irvine Scientific, Santa Ana, California, USA] 


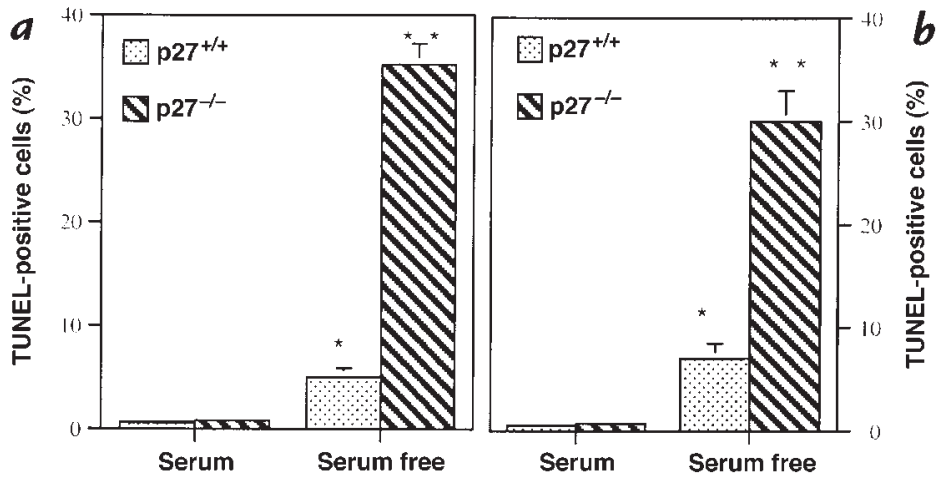

for mouse cells; RPMI for rat cells) plus FCS (Summit Biotechnology, Ft. Collins, Colorado, USA; 20\% for mouse mesangial cells and $10 \%$ for rat mesangial cells and fibroblasts) and allowed to adhere overnight. To induce apoptosis, growth media were removed, cells were washed three times with HBSS, and the medium was replaced with serum-free media (growth media without FCS). In rat mesangial cells and rat fibroblasts, apoptosis was measured (see below) before growth factor withdrawal and at 6,10 , and $20 \mathrm{~h}$ after serum starvation. In $\mathrm{p} 27^{+/+}$ and $\mathrm{p} 27^{-/-}$mesangial cells and fibroblasts, apoptosis was measured before growth factor withdrawal and $24 \mathrm{~h}$ after serum starvation. Apoptosis was also measured at day 5 of serum starvation by simple visual inspection.

In separate experiments, apoptosis was measured in transfected rat mesangial cells and $\mathrm{p} 27^{-/-}$and $\mathrm{p} 27^{+/+}$mesangial cells grown for $24 \mathrm{~h}$ in growth media with cycloheximide $(50 \mu \mathrm{M}$; Sigma Chemical Co., St. Louis, Missouri, USA). All experiments were performed a minimum of four times.

Measuring apoptosis and DNA synthesis. Apoptosis was measured by terminal deoxynucleotide transferase-mediated nick end-labeling (TUNEL) staining and staining with hematoxylin and eosin (H\&E) and Hoechst 33342(Sigma Biosciences, St. Louis, Missouri, USA). For TUNEL and H\&E staining, the medium was carefully removed to collect any detached cells ("floaters"). Adherent cells were scraped off the plate and added to the collected medium, pelleted, resuspended, and fixed in $10 \%$ formalin for $3 \mathrm{~h}$. A cell smear was prepared on a glass slide and air dried. TUNEL staining was performed as described previously (18), and the percentage of TUNEL-positive cells was measured on 200 consecutive cells. Cell morphology was determined by $\mathrm{H} \& \mathrm{E}$ staining. Cells were grown on plastic dishes for Hoechst staining, and at each time point Hoechst was added to the media for $5 \mathrm{~min}$ before quantitation. Apoptosis was defined as the presence of nuclear condensation on Hoechst staining. To ensure that apoptosis was independent of proliferation, DNA synthesis was measured at $24 \mathrm{~h}$ in growth factor-enriched and -deprived $\mathrm{p}^{27^{+/+}}$and $\mathrm{p} 27^{-/-}$cells by BrdU staining as reported elsewhere (6).

Transfection and double-immunostaining. To determine if reconstituting p27 rescued cells from apoptosis, p27-/- mesangial cells and $\mathrm{p} 27^{-/-}$fibroblasts were transiently transfected with plasmids containing the human wild-type p27 DNA (0.005 $\mu \mathrm{g} / \mu \mathrm{l})$. To show specificity for $\mathrm{p} 27$ in rescuing cells from apoptosis, control cells were transfected with plasmids containing mutant $\mathrm{p} 27$ that is incapable of binding CDK2 or $\beta$-galactosidase (concentration of each plasmid was $0.005 \mu \mathrm{g} / \mu \mathrm{l}$ ). Plasmids were complexed with Superfect Transfection Reagent (QIAGEN Inc., Valencia, California, USA). To ensure that p27 expression was reconstituted, Western blot analysis was performed on $20 \mu \mathrm{g}$ of total protein with an antibody to p27 (Santa Cruz Biotechnology Inc., Santa Cruz, California, USA) as reported elsewhere (20).

\begin{abstract}
Figure 1
Quantitation of apoptosis. TUNEL staining was not increased in the presence of growth factors (serum) in p27 ${ }^{-/-}$mesangial cells $(\boldsymbol{a})$ and $\mathrm{p} 27^{-/-}$fibroblasts $(\boldsymbol{b})$. In contrast, TUNEL staining was increased at $24 \mathrm{~h}$ of growth factor deprivation (serum free) in p27-/- cells compared with $\mathrm{p} 27^{+/+}$cells. ${ }^{*} P<0.001 \mathrm{vs}$. serum; ${ }^{*} P$ $<0.001$ vs. $\mathrm{p} 27^{+/+}$. TUNEL, deoxynucleotide transferase-mediated nick end-labeling.
\end{abstract}

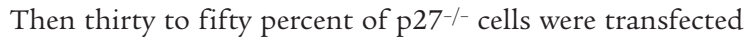
with the plasmids listed above, as measured by staining for green fluorescent protein (GFP) and $\beta$-galactosidase. Thus, to determine if transfection altered apoptosis, $\mathrm{p} 27^{-/-}$mesangial cells and p27//- fibroblasts were cotransfected with GFP plasmid (green nuclei) and double-stained with Hoechst (blue nuclei); the percentage of transfected cells undergoing apoptosis (bright, condensed nuclei) was measured in 200 cells. Similar studies were performed with p27-/- cells cotransfected with GFP and the mutant p27 plasmid that is incapable of binding CDK2. In sep-

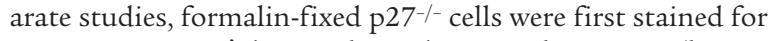
TUNEL using 3,3'-diaminobenzidine as a chromogen (brown nuclei) and then double-stained with an antibody to human p27 (Santa Cruz Biotechnology Inc.) using a fluorescent detection system (green nuclei); the percentage of transfected cells undergoing apoptosis was measured in 200 cells.

Histone H1 kinase assay and CDK2 inhibition. To measure CDK2 activity, $250 \mu \mathrm{g}$ of total protein lysates from $\mathrm{p} 27^{-/-}$and $\mathrm{p} 27^{+/+}$ cells were immunoprecipitated with antibodies to CDK2, cyclin $\mathrm{E}$ and cyclin A (antibodies from Santa Cruz Biotechnology) for $60 \mathrm{~min}$ at $4^{\circ} \mathrm{C}(20)$. Negative controls included omitting the primary antibody, substituting preimmune rabbit serum for primary antibodies, and peptide absorption; positive controls included recombinant cyclin E-CDK2 and cyclin A-CDK2 (20). Antibody specificity was also ensured by repeating the studies with antibodies to CDK2, cyclin E, and cyclin A from a different source (laboratory of J.M. Roberts). CDK2 activity was quantitated by measuring ${ }^{32} \mathrm{P}$ incorporation of individual bands cut out from the gel in a scintillation counter as described previ-
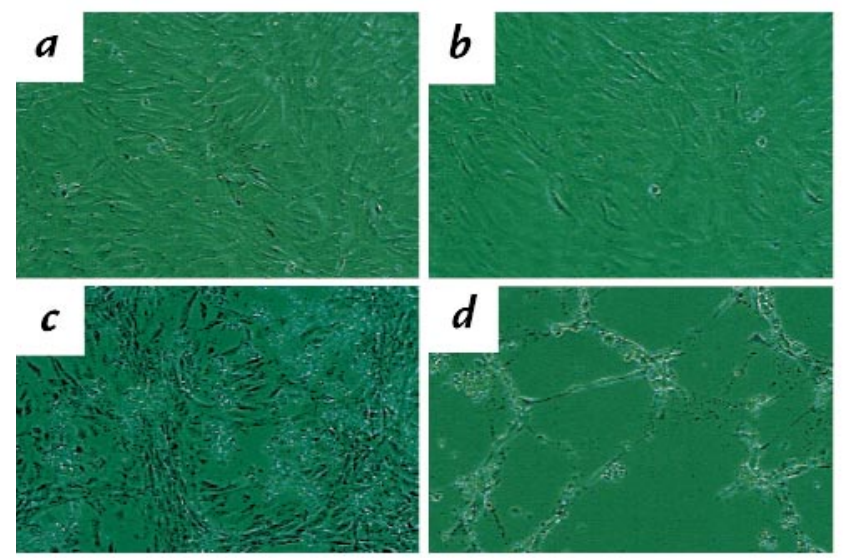

\section{Figure 2}

Effect of growth factor deprivation on cell confluency. p27 $27^{+/+}(a)$ and p27-/- $(\boldsymbol{b})$ mesangial cells were plated at the same density in FCS. Compared with $\mathrm{p} 27^{+/+}$mesangial cells $(\boldsymbol{c})$ cell confluency decreased in $\mathrm{p} 27^{-1-}$ mesangial cells $(\boldsymbol{d})$ after 5 days of growth factor deprivation. 

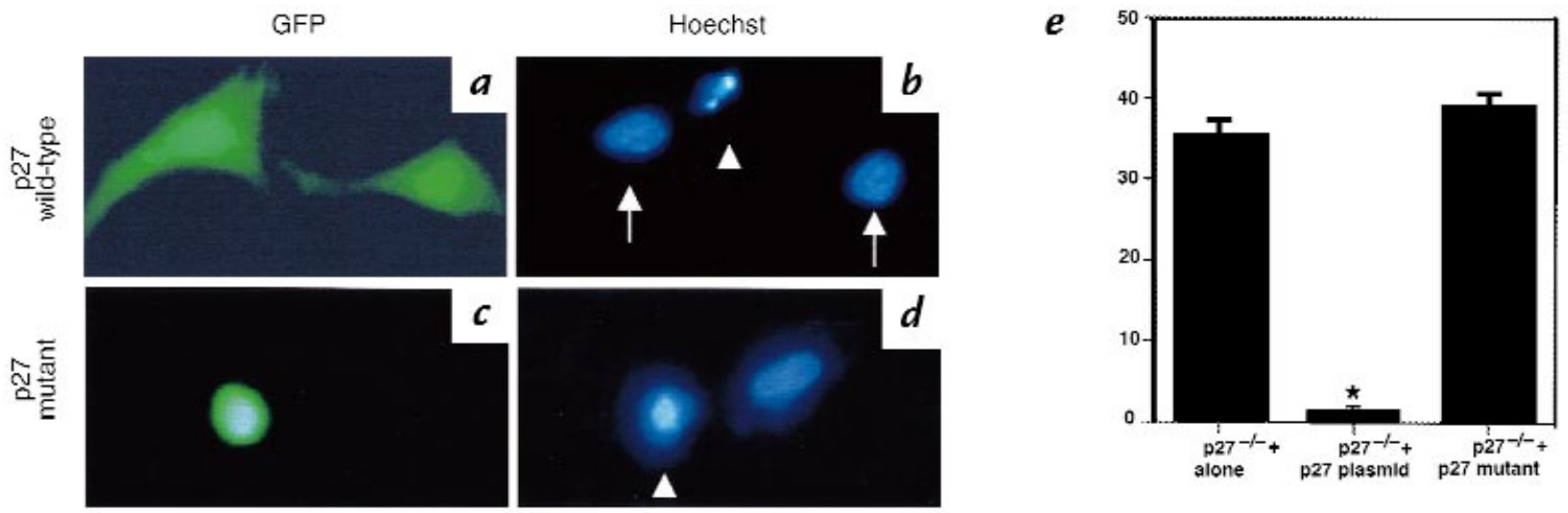

Figure 3

Effect of reconstituting p27 on apoptosis in p27-/- mesangial cells. Transfected p27 mesangial cells were identified by staining with GFP (green nuclei), and apoptosis was measured by Hoechst staining. (a) p27-/- mesangial cells transfected with human p27 plasmid stained green. (b) Apoptosis was not detected in serum-starved transfected p27-/-cells transfected with the p27 plasmid (arrows); apoptosis was detected in nontransfected cells (arrowhead). (c) Control p27-/- mesangial cells were transfected with a p27 mutant plasmid that does not bind CDK2 and GFP. (d) p27 mutant plasmid did not prevent apoptosis (arrowhead). (e) In each experiment, 200 cells were quantitated, and the number of cells undergoing apoptosis was expressed as a percentage. ${ }^{*} P<0.001$ vs. p27-/- alone (nontransfected cells) and control ( 27 mutant plasmid-transfected) p27-/- cells. These data show that p27 protects cells from survival and mitogenic growth factor deprivation-induced apoptosis. CDK2, cyclin-dependent kinase-2; GFP, green fluorescent protein

ously. The background activity was subtracted, as was the activity from a reagent blank. Densitometry was also used to measure CDK2 activity as reported elsewhere.

To ensure that the changes in CDK2 activity were not due to differences in protein levels for CDK2, cyclin E, and cyclin A, Western blot analysis using the antibodies listed earlier was performed.

To determine the role of CDK2 in apoptosis induced by growth factor withdrawal, p27-/- cells were cotransfected with a dominant-negative mutant CDK2 plasmid (dnk2; 0.005 $\mu \mathrm{g} / \mu \mathrm{l}$ ) and GFP or control wild-type CDK2 plasmid (wtk2; $0.005 \mu \mathrm{g} / \mu \mathrm{l}$ ) and GFP. Double-immunostaining was performed with Hoechst to determine the percentage of transfected (green) cells undergoing apoptosis (nuclear condensation), and apoptosis was measured in 200 consecutive cells.

Statistics. Statistical analysis was performed using Statview 4.5 software program (Abacus Concepts, Berkeley, California, USA); statistical significance was evaluated using the Student's $t$ test. A modified $t$ test was performed using Bonferroni-Dunn correction.

\section{Results}

Proliferation requires the presence of growth factors in $\mathrm{p}^{27^{-1-}}$ cells. Bromodeoxyuridine (BrdU) staining, a marker of DNA synthesis, was increased in $\mathrm{p}^{27^{-/-}}$mesangial cells $(27 \pm 7 \%$ vs. $16 \pm 5 \% ; P<0.05)$ and fibroblasts $(25 \pm 6 \%$ vs. $15 \pm 4.8 \% ; P<0.05)$ compared with $\mathrm{p} 27^{+/+}$cells when exposed to FCS for 24 hours. In contrast, BrdU staining was barely detected in $\mathrm{p}^{27^{-/-}}$mesangial cells $(3 \pm 0.3 \%$ vs. $2 \pm 0.4 \% ; P>0.05)$ and fibroblasts $(2.1 \pm 0.4 \%$ vs. $1.9 \pm$ $0.5 \% ; P>0.05)$ deprived of growth factors for 24 hours compared with $\mathrm{p}^{27^{+/+}}$cells. In this study (results not shown) and in a previous report (6), BrdU staining was not detected in rat mesangial cells transfected with p27 antisense in the absence of growth factors $(P>0.05$ vs. nontransfected cells; cells transfected with mismatch). Thus, in the absence of growth factors, the loss of p27 was not sufficient to increase DNA synthesis.

Apoptosis is increased in growth factor-deprived $p 27^{-/-}$cells. Mesangial cells and fibroblasts from p27 null (p27-/-) and $\mathrm{p} 27$ wild-type $\left(\mathrm{p}^{27^{+/+}}\right)$mice were grown in the pres- ence or absence of serum, a source of growth and survival factors, for 24 hours, and the percentage of apoptotic cells was determined by TUNEL staining. In the presence of serum, there were very few TUNEL-positive cells, and there was no significant effect of p27 deficiency (p27-/vs. $\mathrm{p}^{27^{+/+}}$mesangial cells: $0.6 \pm 0.1 \%$ vs. $0.8 \pm 0.1 \%, P>$

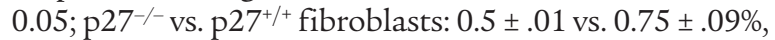
$P>0.05$ ) (Fig. 1). In contrast, when deprived of growth factors for 24 hours, there was a marked increase in TUNEL staining in $\mathrm{p}^{27^{-/-}}$compared with $\mathrm{p} 27^{+/+}$mesangial cells $(35.2 \pm 2 \%$ vs. $5.0 \pm 0.8 \% ; P<0.001)$ and $\mathrm{p} 27^{-/}$ compared with $\mathrm{p}^{27^{+/+}}$fibroblasts $(30 \pm 3 \%$ vs. $7.0 \pm 1.5 \%$; $P<0.001)$ (Fig. 1). Similar results were obtained when apoptotic cells were scored by other methods, including $\mathrm{H} \& \mathrm{E}$ and Hoechst staining. At later time points, the increased death of $\mathrm{p} 27$-deficient cells could be detected by simple visual inspection. Thus, $\mathrm{p}^{27^{-/-}}$and $\mathrm{p}^{27^{+/+}}$ mesangial cells and fibroblasts were plated at the same confluency, allowed to adhere overnight, and then serum starved for five days. There was a marked decrease in p $27^{-/-}$mesangial cell confluency compared with $\mathrm{p} 27^{+/+}$ cells (Fig. 2), and this was associated with an increase in the number of detached cells, or floaters (not shown).

To determine if $\mathrm{p} 27$ also protected cells from other forms of apoptosis, $\mathrm{p}^{27^{+/+}}$and $\mathrm{p} 27^{-/-}$mesangial cells were exposed to the protein synthesis inhibitor cycloheximide for 24 hours. Cycloheximide increased apoptosis in $\mathrm{p}^{27^{-/}}$mesangial cells compared with control cells $(29.2 \pm 3.8 \%$ vs. $14.19 \pm 1.9 \%, P<0.05)$. These results showed that augmented apoptosis in p27-deficient cells may be a generalized phenomenon.

Reconstituting $p 27$ rescues cells from apoptosis. We next determined if $\mathrm{p} 27^{-/-}$mesangial cells and $\mathrm{p} 27^{-/-}$fibroblasts were protected from apoptosis by transient expression of the p27 protein. Cells transfected with the p27 expression vector were identified by cotransfection with a marker plasmid encoding the GFP plasmid, and apoptosis was induced by serum starvation for 24 hours. In 

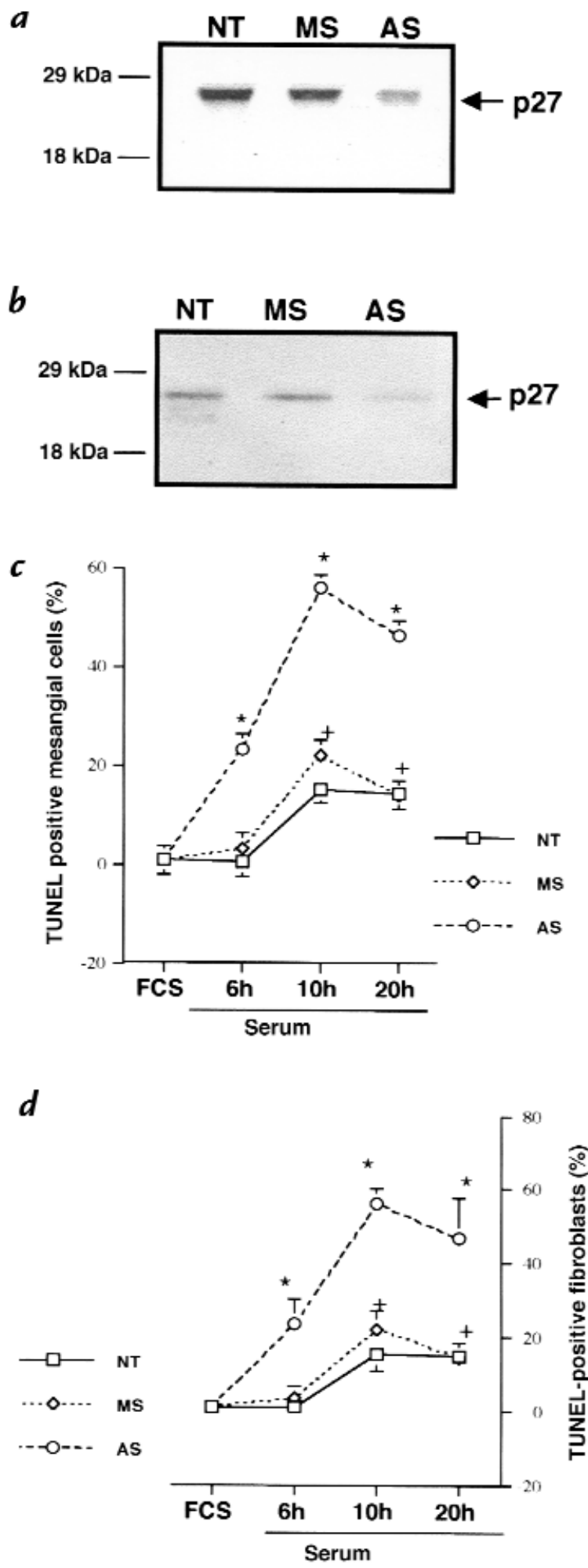

Figure 4

( $\boldsymbol{a}$ and $\boldsymbol{b}$ ) Western blot analysis for $\mathrm{p} 27$. Transfecting rat mesangial cells $(a)$ and rat fibroblasts $(b)$ with antisense oligonucleotides to p27 (AS), but not mismatch oligonucleotides (MS), lowered p27 protein levels compared with nontransfected cells $(N T)$. ( $\boldsymbol{c}$ and $\boldsymbol{d}$ ) Quantitation of TUNEL staining in rat cells. TUNEL staining was detected at $6 \mathrm{~h}$ after growth factor deprivation (serum free) in AS-transfected cells in rat mesangial cells $(c)$ and rat fibroblasts $(d)$, but not in controls, and was increased at each time point compared with controls. separate experiments, apoptosis was measured in $\mathrm{p}^{27^{-/-}}$ transfected cells by double-immunostaining for the p27 antigen and TUNEL. Apoptosis $\left(\mathrm{GFP}^{+} /\right.$Hoechst $\left.{ }^{+}\right)$was not detected in $200 \mathrm{p} 27^{-/-}$mesangial cells and $\mathrm{p}^{27^{-/-}}$ fibroblasts transfected with the $\mathrm{p} 27$ expression vector when scored 24 hours after serum starvation (Fig. 3). Similar results were obtained using double-staining for p27 and TUNEL (p27 $/$ TUNEL $^{+}$) (not shown).

To test specificity for the transfection studies with p27 wild-type plasmid discussed above, similar studies were performed in $\mathrm{p}^{27^{-/}}$cells transfected with a p27 mutant plasmid that cannot bind to CDK2. In contrast to p27 wild-type plasmid, transfecting $\mathrm{p}^{27^{-/-}}$cells with plasmids expressing $\mathrm{p} 27$ mutant protein that is incapable of binding CDK2 (Fig. 3), $\beta$-galactosidase (not shown) or vector alone (not shown) did not rescue $\mathrm{p} 27^{-/-}$cells from apoptosis. Furthermore, the rate of apoptosis was comparable in nontransfected $\mathrm{p} 27^{-/-}$cells and $\mathrm{p} 27^{-/-}$cells transfected with the p 27 mutant plasmid. These results showed that reconstituting p27 reduced apoptosis in p27 $7^{-/}$cells to a level comparable to that of control cells, and also confirmed the specificity of p27 wild-type plasmid in protecting cells from apoptosis.

p27 also protects rat cells from apoptosis. To determine if p27 also protected cells from other species from apoptosis, p27 levels were lowered in rat mesangial cells and rat fibroblasts with antisense oligodeoxynucleotides to p27 $(6,20)$ and then deprived of growth factors for 6,10 , and 20 hours. Controls included nontransfected cells and cells transfected with mismatch oligonucleotides (Fig. 4). The exclusion of toluidine blue and the absence of released lactate dehydrogenase showed that transfecting these cells did not alter cell viability (results not shown). In the presence of serum, apoptosis was not detected in transfected rat mesangial cells and fibroblasts (Fig. 4). In contrast, apoptosis was significantly increased in serumstarved rat mesangial cells and rat fibroblasts transfected with p27 antisense in comparison to the controls (Fig. 4). These results showed that p27 levels modulate the degree of apoptosis in cells from different species.

CDK2 activity is increased in growth factor-deprived $p 27^{-/-}$ cells. As shown earlier, DNA synthesis as measured by BrdU staining was barely detected in mesangial cells and fibroblasts from $\mathrm{p} 27^{-/-}$and $\mathrm{p} 27^{+/+}$mice after withdrawal of growth factors at 24 hours. However, p27 deficiency did have a measurable effect on regulation of CDK2 by mitogens. Fig. 5 shows that CDK2 kinase activity was increased in $\mathrm{p}^{27^{-/}-}$mesangial cells and $\mathrm{p}^{27^{-/-}}$fibroblasts in the presence of growth factors (serum) compared with $\mathrm{p}^{27^{+/+}}$cells. Eighteen hours of serum starvation reduced CDK2 activity to very low levels in $\mathrm{p}^{27^{+/+}}$cells. In contrast, CDK2 activity was readily detected in serum-starved $\mathrm{p}^{27^{-/-}}$cells at the same time point (Fig. 5). CDK2 activity was not detected when the primary antibody was omitted or when the primary antibody was absorbed with peptide (results not shown). Furthermore, the increase in CDK2 activity was also shown with a different CDK2 antibody from a different source. In contrast to changes in CDK2 activity, the protein levels for CDK2 remained unchanged in $\mathrm{p}^{27^{-/-}}$and $\mathrm{p}^{27^{+/+}}$cells in the presence or absence of serum (Fig. 5). These results are 


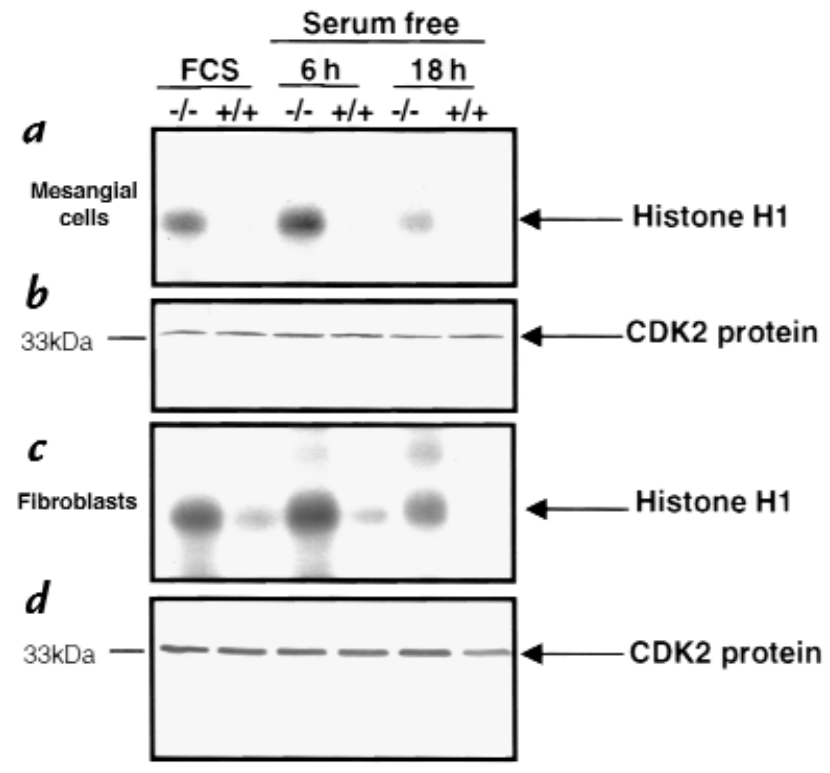

Figure 5

CDK2 activity and protein levels. Total cell protein was immunoprecipitated with an antibody to CDK2, and CDK2 activity was detected by $\mathrm{H} 1$ kinase assay. CDK2 protein levels were measured by Western blot analysis. In the presence of survival and mitogenic growth factors (FCS), CDK2 activity was increased in p $27^{-/-}$mesangial cells $(\boldsymbol{a})$ and $\mathrm{p} 27^{-/-}$fibroblasts (c) compared with $\mathrm{p} 27^{+/+}$cells. CDK2 activity remained elevated at $6 \mathrm{~h}$ and $18 \mathrm{~h}$ after growth factor deprivation (serum free) in $\mathrm{p} 27^{-/-}$cells compared with $p 27^{+/+}$cells. The protein levels for CDK2 did not change in $\mathrm{p} 27^{-/-}$and $\mathrm{p} 27^{+/+}$mesangial cells $(\boldsymbol{b})$ and fibroblasts $(\boldsymbol{d})$ in the presence or absence of growth factors.

likely to represent a delay in the downregulation of CDK2 in the absence of $\mathrm{p} 27$, rather than differences in CDK2 protein levels.

Uncoordinated CDK2 activity in apoptotic cells. To determine if the unconstrained CDK2 activity in growth factor-deprived $\mathrm{p} 27^{-/-}$cells represented cyclin E or cyclin A activation, immunoprecipitation studies were performed with antibodies to these cyclins. Fig. 6 shows that in p27-/- cells the presence of growth factors was associated with an increase in cyclin E-CDK2 activity and cyclin A-CDK2 activity measured by ${ }^{32} \mathrm{P}$ incorporation and densitometry. However, in $\mathrm{p}^{27^{-/-}}$mesangial cells deprived of growth factors for six hours, there was a $47 \%$ decrease in cyclin $\mathrm{E}$ activity (Fig. 6). In contrast, despite the absence of growth factors for six hours, cyclin A-CDK2 activity remained elevated in $\mathrm{P}^{27^{-/}}$mesangial cells (Fig. 6).

To determine if the increase in cyclin A-CDK2 activity (but not cyclin E-CDK2 activity) also occurred in growth factor-deprived $\mathrm{p} 27^{+/+}$mesangial cells, immunoprecipitation studies were performed. There was a $45 \%$ decrease in cyclin $\mathrm{E}$ activity and a $43 \%$ decrease in cyclin A activity after six hours of serum deprivation in $\mathrm{p} 27^{+/+}$cells compared with cells exposed to growth factors (Fig. 6). Furthermore, the decrease in cyclin E-CDK2 activity with growth factor deprivation was similar in $\mathrm{p}^{27^{-/-}}$and $\mathrm{p} 27^{+/+}$mesangial cells. In contrast, there was almost a twofold increase in cyclin A-CDK2 activity in growth factor-deprived $\mathrm{p} 27^{-/-}$cells compared with growth factor-deprived $\mathrm{p} 27^{+/+}$cells.
To determine if the difference in CDK2 kinase activity in $\mathrm{p} 27^{-/-}$and $\mathrm{p} 27^{+/+}$mesangial cells was due to differences in protein levels for CDK2, cyclin E, or cyclin A, Western blot analyses were performed for these proteins. Fig. 5 shows that the protein levels for CDK2 remained constant in $\mathrm{p} 27^{-/-}$and $\mathrm{p} 27^{+/+}$mesangial cells in the presence or absence of growth factors. Furthermore, the protein levels for cyclin E and cyclin A were similar in p27 $7^{-/}$and $\mathrm{p} 27^{+/+}$ cells in the presence of serum, and the levels of these cyclins was similar in $\mathrm{p} 27^{-/-}$and $\mathrm{p} 27^{+/+}$cells deprived of growth factors for six hours (not shown). These findings show that the increase in cyclin A-CDK2 activity in apoptotic p27-/- cells was not due to the levels of specific cyclins and CDK2, but rather due to the absence of p27.

Thus, when compared with absent CDK2 activity in normal cells, CDK2 activity in p27-deficient cells was transiently elevated after mitogen withdrawal, and this was due to cyclin A-CDK2. However, the elevated CDK activity was accompanied not by DNA synthesis (absent BrdU staining), but rather by cell death.

p27 limits apoptosis by restraining CDK2. Our results suggested that CDK2 was the effector for apoptosis in this model and that $\mathrm{p} 27$ protected cells from apoptosis by maintaining CDK2 inactive. To test this hypothesis, p27-/cells were first exposed to the purine analogue Roscovitine, which reduces CDK2 activity $(21,22)$. When p27-/- cells were incubated in serum-free media and $12.5 \mu \mathrm{M}$ Roscov-
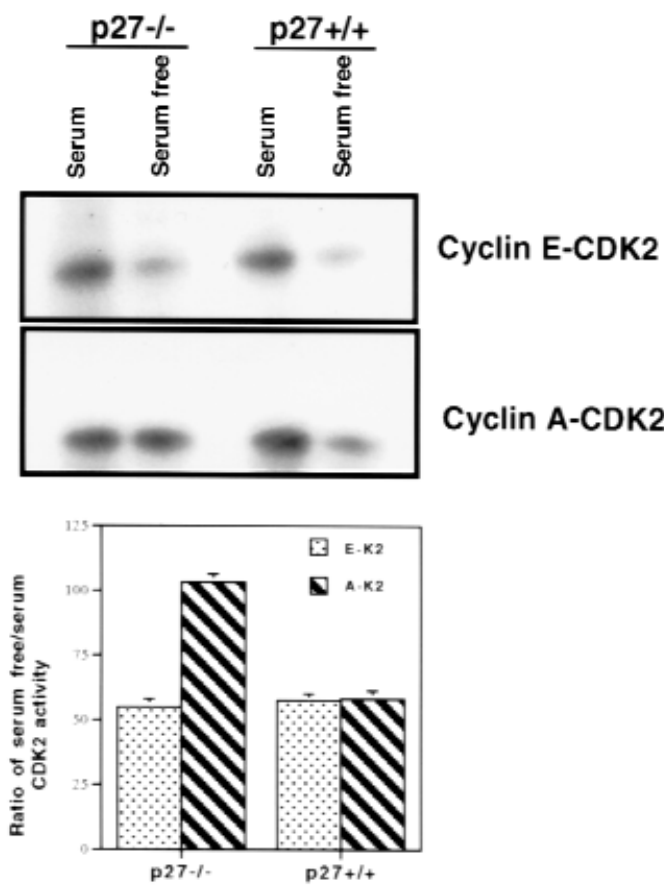

Figure 6

Cyclin E-CDK2 and cyclin A-CDK2 activity in p27-/- and $\mathrm{p} 27^{+/+}$mesangial cells. Total protein was extracted from $\mathrm{p} 27^{-/-}$and $\mathrm{p} 27^{+/+}$mesangial cells and immunoprecipitated with antibodies to cyclin E (top) and cyclin A (middle) for histone $\mathrm{H} 1$ kinase. Quantitation of CDK2 kinase activity is shown (bottom). In the presence of growth factors (serum), there was an increase in cyclin E-CDK2 and cyclin A-CDK2 activity in p27-/- and p27 $27^{+/+}$ cells, which decreased in $\mathrm{p} 27^{+/+}$cells after $6 \mathrm{~h}$ of growth factor deprivation (serum free). In p27-/- mesangial cells, cyclin A-CDK2 activity, but not cyclin E-CDK2 activity, remained increased after growth factor deprivation. 

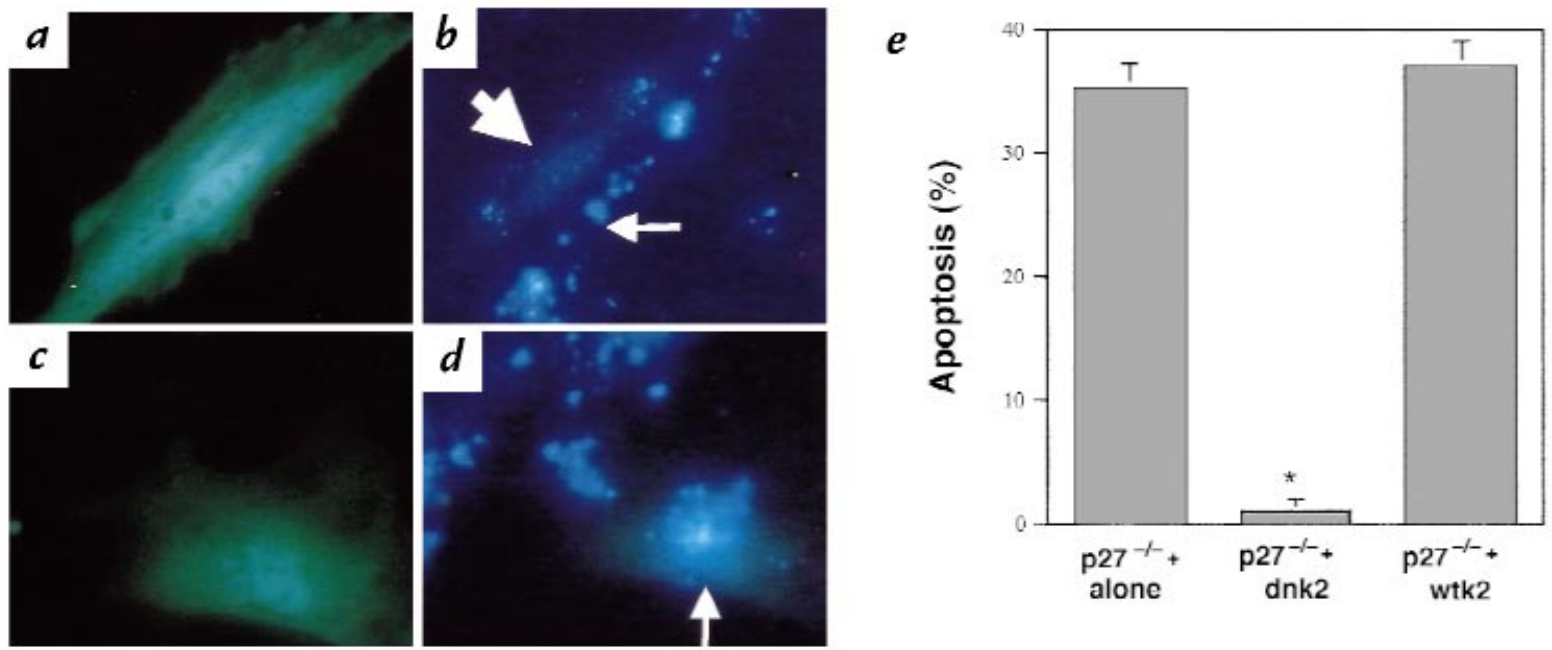

Figure 7

Effect of suppressing CDK2 activity on apoptosis in p27-/- fibroblasts in response to serum starvation. (a) p27-/- cells transfected with a dominant-negative mutant CDK2 (dnk2) plasmid were identified by cotransfection with a GFP plasmid (green nuclei). ( $\boldsymbol{b}$ ) Apoptosis (measured by Hoechst staining) was not detected in dnk2-transfected cells (thick arrow), but was detected in nontransfected cells (thin arrow). (c) p27 ${ }^{-/-}$cells cotransfected with wild-type CDK2 (wtk2) plasmid and GFP were identified as green. (d) wtk2 did not protect p27 ${ }^{-/-}$fibroblasts from apoptosis (arrow). (e) Quantitation of apoptosis. The percentage of apoptotic cells was evaluated in 200 cells. ${ }^{*} P<0.001$ vs. nontransfected $p 27^{-1-}$ fibroblasts ( $227^{-/-}$alone) and wtk2-transfected cells. Similar results were obtained in $\mathrm{p} 27^{-/-}$mesangial cells.

itine for 24 hours, apoptosis was reduced compared with cells exposed to serum-free media and dimethyl sulfoxide ( $3.5 \pm 0.9 \%$ vs $34 \pm 2 \% ; P<0.001)$. Second, $\mathrm{p}^{27^{-/}}$mesangial cells and $\mathrm{p} 27^{-/-}$fibroblasts were cotransfected with a dominant-negative mutant CDK2 plasmid ( $\mathrm{dnk} 2)$ and a plasmid expressing GFP, or the control wild-type CDK2 and GFP, and then deprived of growth factors for 24 hours. The percentage of $\mathrm{p}^{27^{-/-}}$cells undergoing apoptosis after serum starvation was not reduced by overexpression of wild-type CDK2 (Fig. 7) or an irrelevant plasmid ( $\beta$-galactosidase results not shown). In striking contrast, no apoptosis was detected in cells overexpressing kinase inactive CDK2 (0/200 GFP-positive cells) (Fig. 7). These results show that inhibiting CDK2, either pharmacologically or by overexpression of a kinase inactive mutant CDK2, significantly suppresses apoptosis in $\mathrm{p}^{27^{-/-}}$cells.

\section{Discussion}

In this study, we show that apoptosis, but not proliferation, is increased in mesangial cells and fibroblasts when the levels of the CDK inhibitor p27 are absent or reduced. Reconstituting p27 rescued cells from apoptosis, showing that a novel function of $\mathrm{p} 27$ is to modulate apoptosis. The loss of $\mathrm{p} 27$ was associated with increased cyclin A-CDK2 activity, but not cyclin E-CDK2 activity, and inhibiting CDK2 protected cells from apoptosis, showing that CDK2 was the death effector in $\mathrm{p} 27^{-/-}$cells.

The known biological functions of the CDK inhibitor p27 are closely linked with its protein levels. Thus, it has been described that high p27 levels are required to maintain cells in quiescence (4), to control the restriction point in the cell cycle (20), and to promote cell-cycle exit in response to antimitogenic cues (23) and differentiation (2). Thus, proliferation requires that $\mathrm{p} 27$ levels be lowered, and lowering p27 levels with antisense augments proliferation induced by mitogenic growth factors $(6,20)$.
Thus, the conventional view is that the role for p27 is determining the proliferative threshold to mitogenic growth factors. However, in this study, we show that a loss of $\mathrm{p} 27$, in the absence of a complete mitogenic signal, was not sufficient to cause cells to enter the $S$ phase of the cell cycle. Thus, proliferation requires both a loss of p27 and the presence of growth factors.

Previous studies have shown that proliferation and apoptosis are closely linked (18). The first major finding in this study was that $\mathrm{p} 27$ protects mesangial cells and fibroblasts from apoptosis induced by growth factor withdrawal. This occurred independent of proliferation.

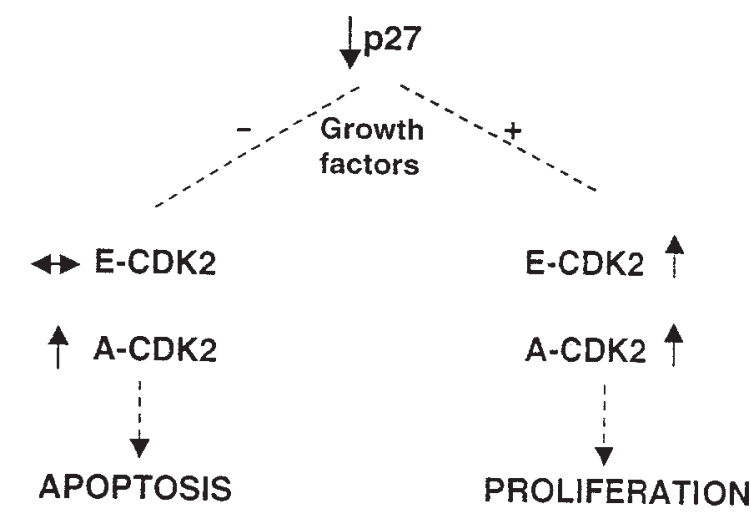

\section{Figure 8}

Proposed schema showing coordination of cell-cycle events by the CDK inhibitor p27. When grown in the presence of growth factors, reduced or absent p27 levels are associated with a coordinated and synchronous increase in cyclin E-CDK2 and cyclin A-CDK2 activity. This favors cellcycle progression and proliferation. Under stress states such as growth factor deprivation, the loss of $\mathrm{p} 27$ is associated with an unconstrained increase in cyclin A-CDK2 activity, but not cyclin E-CDK2 activity. This unscheduled increase in CDK2 activity causes cell-cycle exit by apoptosis. 
In the presence of growth factors, apoptosis was barely detected in normal and $\mathrm{p} 27^{-/-}$cells. In contrast, apoptosis was markedly increased in growth factor-deprived p27 $7^{-/-}$cells compared with normal cells. Furthermore, the onset of apoptosis induced by serum starvation occurred earlier in cells with absent or reduced p27 levels compared with normal cells. Moreover, reconstituting p27 levels by transient transfection protected $\mathrm{p}^{27^{-/-}}$cells from apoptosis. The specificity for $\mathrm{p} 27$ was confirmed by the failure of the mutant p27 plasmid that cannot bind CDK2 to protect against apoptosis. The increase in apoptosis in p27-deficient cells was shown in different cell types and different species in response to two well-described inducers of apoptosis. These results show that a new biological function for p27 is to modulate apoptosis induced by growth factor withdrawal and cycloheximide.

Mesangial cell apoptosis and proliferation are increased in many types of glomerular diseases $(24,25)$, and apoptosis is critical for the resolution phase following glomerular injury in immune-mediated glomerulonephritis (18). The decrease in p27 levels in experimental glomerulonephritis coincides with mesangial cell proliferation and apoptosis (8). There is also a greater amount of apoptosis

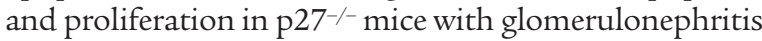
compared with controls (9). Thus, in this study, we specifically chose growth factor deprivation to induce apoptosis (26). Our results showed that apoptosis, not proliferation, was increased in growth factor-deprived $\mathrm{p} 27^{-/-}$cells compared with normal cells. These results show that when p27 levels are reduced or absent, cells successfully progress through the cell cycle and proliferate in the presence of growth factors. In contrast, as shown in this study, under stress states such as growth factor deprivation, cells with reduced or absent p27 levels exit the cell cycle and undergo an apoptotic or programmed cell death. Furthermore, adding back p27 to p27-deficient ( $\mathrm{p}^{27^{-/}}$) cells to restore the levels of the CDK inhibitor abrogates the increase in apoptosis. Moreover, transfecting the mutant p27 that cannot bind CDK2 did not protect $\mathrm{p}^{27^{-/}}$cells from apoptosis, thereby confirming the specificity for $\mathrm{p} 27$ in rescuing $\mathrm{p}^{27^{-/}}$cells from apoptosis. These results show that apoptosis and proliferation are governed independently by the levels of the CDK inhibitor p27.

There is an increasing body of evidence showing that CDK inhibitors safeguard against apoptosis (27-30). Within the Cip/Kip family of CDK inhibitors, p21 Cip1,Waf1 and $\mathrm{p} 57^{\mathrm{Kip} 2}$ have been shown to limit apoptosis $(31,32)$. Furthermore, it has also been shown that $\mathrm{p} 21^{\text {Cip1,Waf1 }}$ and p27 are cleaved during growth factor deprivation-induced apoptosis in endothelial cells (33). However, this study is the first to provide evidence that the third member (p27) of the Cip/Kip family of CDK inhibitors also protects cells from apoptosis.

One effect of p27 deficiency appears to be a transient uncoupling of CDK downregulation from the receipt of an antiproliferative signal. In some cases, this seems to result in an extra round of cell division $(20,34)$. But, as shown here, this may also lead to cell death. The second major finding in this study was that despite the absence of mitogenic growth factors, CDK2 activity remained elevated in $\mathrm{p}^{27^{-/-}}$cells compared with normal cells. The increase in CDK2 activity in growth fac- tor-deprived cells was not due to differences in CDK2 protein levels, which remained unchanged in the presence or absence of growth factors. Moreover, inhibiting CDK2 either pharmacologically or with a dominant-negative mutant protected $\mathrm{p} 27^{-/-}$from apoptosis. These results demonstrate that CDK2 was the death effector in this model.

Previous studies have shown that growth factor stimulation leads to a coordinated and synchronous activation of CDK2 in a cell cycle-dependent manner. Thus, CDK2 is activated by cyclin $\mathrm{E}$ in late $\mathrm{G} 1$ phase $(35,36)$ and by cyclin A in the S and G2 phases of the cell cycle (37). In this study, we showed that growth factors were associated with increased cyclin E- and cyclin A-CDK2 activity. In contrast, we also showed that $\mathrm{p} 27^{-/-}$cells deprived of growth factors were associated with an uncoordinated or unscheduled activation of CDK2. In serum-starved $\mathrm{p} 27^{-/-}$cells, cyclin E-CDK2 activity was decreased, while cyclin A-CDK2 was markedly increased. In contrast, cyclin E- and cyclin A-CDK2 activity was decreased in normal cells deprived of growth factors. The increase in cyclin A-CDK2 activity in growth factor-deprived $\mathrm{p} 27^{-/-}$cells was not due to differences in protein levels for CDK2, cyclin E, or cyclin A compared with control cells. These results show that the increase in cyclin A-CDK2 activity was due to differences in the levels of the CDK inhibitor p27.

Thus, in serum-starved $\mathrm{p} 27^{-/-}$cells, the increase in cyclin A-CDK2 activity without a preceding and coordinated increase in cyclin E-CDK2 activity may favor cell-cycle exit rather than cell-cycle progression. A role for cyclindependent kinases in apoptosis has been shown $(33,38$, 39). However, here we show that under certain conditions, such as deprivation of growth factors, the inability to constrain CDK2 activity in $\mathrm{p}^{27^{-/-}}$cells is associated with exit from the cell cycle by programmed cell death.

In summary (Fig. 8), this study offers a different perspective on the biological function of the cyclin-dependent kinase inhibitor p27 and suggests that under certain conditions p27 is necessary when cells exit the cell cycle to rapidly constrain CDK activity and thereby prevent cell death. Hence, a more complete view of the function of CDK inhibitors is that they are essential to coordinate the cell-cycle and cell-death programs so that cells can remain viable as they enter a nonproliferative state.

\section{Acknowledgments}

Thanks to Michael Flanagan for p27 oligonucleotides and p27 plasmids, Laurent Meijer for supplying Roscovitine, David M. Hockenberry for the rat-1 fibroblasts, and Q. Qu for technical assistance. This work was supported by National Institutes of Health grants to S.J. Shankland (DK-52121, DK-51096, DK47659) and to J.M. Roberts. J.M. Roberts is an Investigator of the Howard Hughes Medical Institute. K. Hiromura was supported by the Uehara Memorial Foundation.

\footnotetext{
1. Sherr, C.J., and Roberts, J.M. 1995. Inhibitors of mammalian G1 cyclindependent kinases. Genes Dev. 9:1149-1163.

2. Durand, B., Gao, F.-B., and Raff, M. 1997. Accumulation of the cyclindependent kinase inhibitor $\mathrm{p} 27 / \mathrm{Kip} 1$ and the timing of oligodendrocyte differentiation. EMBO J. 16:306-317.

3. Zabludoff, S., Csete, M., Wagner, R., Yu, X., and Wold, B.J. 1998. p27Kip1 is expressed transiently in developing myotomes and enhances myogenesis. Cell Growth Differ. 9:1-11.
} 
4. Nourse, J., et al. 1994. Interleukin-2-mediated elimination of the p27kip1 cyclin-dependent kinase inhibitor prevented by rapamycin. Nature. 372:570-573.

5. Polyak, K., et al. 1994. Cloning of p27Kip1, a cyclin-dependent kinase inhibitor and potential mediator of extracellular antimitogenic signals. Cell. 78:59-66.

6. Shankland, S.J., et al. 1997. Mesangial cell proliferation mediated by PDGF and bFGF is determined by levels of the cyclin kinase inhibitor p27Kip1. Kidney Int. 51:1088-1099.

7. Schoecklmann, H.O., Rupprecht, H.D., Zauner, I., and Sterzel, R.B. 1996 TGF- $\beta 1$ induced cell cycle arrest in renal mesangial cells involves inhibition of cyclin E-CDK2 activation and retinoblastoma protein phosphorylation. Kidney Int. 51:1228-1236.

8. Shankland, S.J., et al. 1996. Changes in cell cycle protein expression during experimental mesangial proliferative glomerulonephritis. Kidney Int. 50:1230-1239.

9. Ophascharoensuk, V., Fero, M.L., Hughes, J., Roberts, J.M., and Shankland, S.J. 1998. The cyclin-kinase inhibitor $\mathrm{p} 27^{\mathrm{Kip} 1}$ safeguards against inflammatory injury. Nat. Med. 4:575-580.

10. Shankland, S.J., et al. 1997. Cyclin kinase inhibitors are increased during experimental membranous nephropathy: potential role in limiting glomerular epithelial cell proliferation in vivo. Kidney Int. 52:404-413.

11. Rosenblatt, J., Gu, Y., and Morgan, D.O. 1992. Human cyclin-dependent kinase 2 is activated during the S and G2 phases of the cell cycle and associates with cyclin A. Proc. Natl. Acad. Sci. USA. 89:2824-2828.

12. Hoang, A.T., Cohen, K.J., Barrett, J.F., Bergstrom, D.A., and Dang, C.V. 1994. Participation of cyclin A in Myc-induced apoptosis. Proc. Natl. Acad. Sci. USA. 91:6875-6879.

13. Meikrantz, W., Gisselbrecht, S., Tam, S.W., and Schlegel, R. 1994. Activation of cyclin A-dependent protein kinase during apoptosis. Proc. Natl. Acad. Sci. USA. 91:3754-3758.

14. Shi, L., et al. 1996. Granzyme B induces apoptosis and cyclin A-associated cyclin-dependent kinase activity in all stages of the cell cycle. $J$. Immunol. 157:2381-2385.

15. Zhou, B.-B., Li, H., Yuan, J., and Kirschner, M.W. 1998. Caspace-dependent activation of cyclin-dependent kinases during Fas-induced apoptosis in Jurkat cells. Proc. Natl. Acad. Sci. USA. 95:6785-6790.

16. Savill, J. 1997. Apoptosis in resolution of inflammation. J. Leukoc. Biol. 61:375-380.

17. Thompson, C.B. 1995. Apoptosis in the pathogenesis and treatment of disease. Science. 267:1456-1462.

18. Baker, A.J., et al. 1994. Mesangial cell apoptosis: The major mechanism for resolution of glomerular hypercellularity in experimental mesangial proliferative nephritis. J. Clin. Invest. 94:2105-2116.

19. Fero, M.L. 1996. A syndrome of multiorgan hyperplasia with features of gigantism, tumorigenesis, and female sterility in p27Kip1-deficient mice. Cell. 85:733-744.

20. Coats S., Flanagan, W.M., Nourse J., and Roberts, J.M. 1996. Requirement of p27Kip1 for restriction point control of the fibroblast cell cycle. Science. 272:877-880.

21. Meijer, L. 1996. Chemical inhibitors of cyclin-dependent kinases. Trends
Cell Biol. 6:393-397.

22. Meijer, L., et al. 1996. Biochemical and cellular effects of roscovitine, a potent and selective inhibitor of the cyclin-dependent kinases cdc2, cdk2 and cdk5. Eur. J. Biochem. 243:527-536.

23. Polyak, K., et al. 1994. p27kip1, a cyclin-Cdk inhibitor, links transforming growth factor- $\beta$ and contact inhibition to cell cycle arrest. Genes Dev. 8:9-22.

24. Shimizu, A., et al. 1995. Apoptosis in the repair process of experimental proliferative glomerulonephritis. Kidney Int. 47:114-121.

25. Tashiro, K., et al. 1998. Detection of apoptotic cells in glomeruli of patients with IgA nephropathy. Nephron. 79:21-27.

26. Eastman, A. 1995. Survival factors, intracellular signal transduction, and the activation of endonucleases in apoptosis. Semin. Cancer Biol. 6:45-52.

27. Wang, J., and Walsh, K. 1996. Resistance to apoptosis conferred by Cdk inhibitors during myocyte differentiation. Science. 273:359-361.

28. Polyak, K., Waldman, T., He, T.C., Kinzler, K.W., and Vogelstein, B. 1996. Genetic determinants of $\mathrm{p} 53$-induced apoptosis and growth arrest. Genes Dev. 10:1945-1952.

29. Poluha, W., et al. 1996. The cyclin-dependent kinase inhibitor p21 (waf1) is required for survival of differentiating neuroblastoma cells. Mol. Cell. Biol. 16:1335-1341.

30. Gorospe, M., et al. 1997. p21 (Waf1/Cip1) protects against p53-mediated apoptosis of human melanoma cells. Oncogene. 14:929-935.

31. Yan, Y., Frisen, J., Lee, M.H., Massague, J., and Barbacid, M. 1997. Ablation of the CDK inhibitor p57Kip2 results in increased apoptosis and delayed differentiation during mouse development. Genes Dev. 11:973-983.

32. Zhang, P., et al. 1997. Altered cell differentiation and proliferation in mice lacking p57Kip2 indicates a role in Beckwith-Wiedermann syndrome. Nature. 387:151-158.

33. Levkau, B., et al. 1998. Cleavage of p21 (Cip1/Waf1) and p27 (Kip1) mediates apoptosis in endothelial cells through activation of CDK2: role of a caspase cascade. Mol. Cell. 1:553-563.

34. Park, D.S., Farinelli, S.E., and Green, LA. 1996. Inhibitors of cyclindependent kinases promote survival of post mitotic neuronally differentiated PC12 cells and sympathetic neurons. J. Biol. Chem. 271:8161-8169.

35. Ohtsubo, M., and Roberts, J.M. 1993. Cyclin-dependent regulation of G1 in mammalian fibroblasts. Science. 259:1908-1912.

36. Ohtsubo, M., Theodoras, A.M., Schumacher, J., Roberts, J.M., and Pagano, M. 1995. Human cyclin E, a nuclear protein essential for the G1to-S phase transition. Mol. Cell. Biol. 15:2612-2624.

37. Girard, F., Strausfeld, U., Fernandez, A., and Lamb, N. 1991. Cyclin A is required for the onset of DNA replication in mammalian fibroblasts. Cell. 67:1169-1179.

38. Meikrantz, W., and Schlegel, R. 1996. Suppression of apoptosis by dominant negative mutants of cyclin-dependent protein kinases. J. Biol. Chem. 271:10205-10209.

39. Harvey, K.J., Blomquist, J.F., and Ucker, D.S. 1998. Commitment and effector phases of the physiological cell death pathway elucidated with espect to Bcl-2, caspace, and cyclin-dependent kinase activities. Mol. Cell. Biol. 18:2912-2922. 